\title{
DESIGN CONCEPT OF TEST STAND FOR DETERMINING PROPERTIES OF MAGNETORHEOLOGICAL ELASTOMERS
}

\author{
Mirosław BOCIAN*, Jerzy KALETA*, Daniel LEWANDOWSKI", Michał PRZYBYLSKI ${ }^{*}$ \\ *Institute of Material Science and Applied Mechanics, Wroclaw University of Technology, \\ ul. Smoluchowskiego 25, 50-370 Wrocław, Poland \\ $\underline{\text { miroslaw.bocian@pwr.wroc.pl, jerzy.kaleta@pwr.wroc.pl, daniel.lewandowski@pwr.wroc.pl, michal.przybylski@pwr.wroc.pl }}$
}

\begin{abstract}
Magnetorheological elastomers (MRE) are "SMART" materials that change their mechanical properties under influence of magnetic field. Thanks to that ability it is possible to create adaptive vibration dampers based on the MRE. To test vibration damping abilities of this material special test stand is required. This article presents design concept for such test stand with several options of testing.
\end{abstract}

Key words: MRE, Vibration Damping, SMART Materials, Halbach Array

\section{INTRODUCTION}

In the modern world there is a great potential for the effective vibration damping. All of us are exposed to vibrations all of the time. Source of vibrations can be anything starting from small electromechanical equipment through all kind of transportation systems and heavy machinery, ending on natural causes like earthquake. All vibrations, regardless its source, can be harmful if they last long enough. The stronger the vibration the smaller time they need to do the damage, for example combustion engines vibrates constantly while vehicle they operate last years undamaged comparing to the Bucket-wheel excavators at openpit mines that frequently get damaged due to strong vibrations they experience.

Group of the modern materials presenting promising properties in terms of vibration damping are magnetorheological elastomers (MRE). Those are materials that change their mechanical properties under influence of external magnetic field (Kaleta et al., 2011). They are categorized as magneto-mechanical materials, often referred as "SMART" magnetic materials. As MRE presents changing mechanical properties depending on magnetic field the test stand for determining their vibration damping properties have to be adjusted to the need of use variable magnetic field.

The article presents the design concept for the test stand for determining damping and stiffness properties of magnetorheological elastomers that can work with several types of excitation and measurement methods.

\section{TEST METHODOLOGY}

The idea of the test stand is based on the one degree of freedom system presented in Fig. 1. Such system is very useful in testing vibration damping as there is just one damping element and all the results refers to it. Thanks to such approach effects not related to tested object can be neglected as they do not interfere with actual results on the frequency response function (FRF).

Except the tested element test stand requires method for exci- tation of the vibrations, response gathering device and signal processor that will collect input and output data of the vibrating test stand. For vibration excitation impact hammer or shaker can be used. In the case of desired test stand impact hammer has advantage over the shaker as it is fast and easy to use technique that does not require variable loading of the system to be applied. The transducer is the universal device used to collect acceleration signal from both impact hammer and shaker excitation. The signal processor have to gather data but also process it in terms of FRF and phase shift angle so the output data will be reliable and easy to analyze. HP 35665A Dynamic Signal Analyzer meets the requirements.

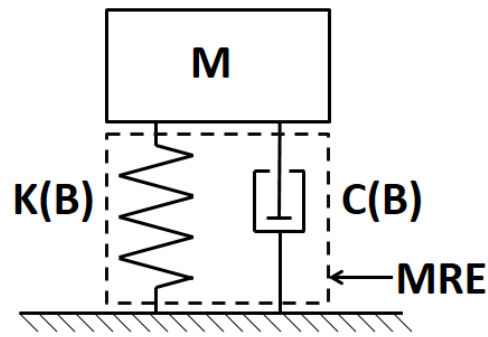

Fig. 1. One degree of freedom system where $K(B)$ and $C(B)$ are the MRE material

Frequency response function is necessary for the analysis of the properties of the system. On its base following parameters can be obtained: damping ratio $\zeta$, structural damping ratio $\eta$, damping $C$ and stiffness $K$. To calculate the damping ratio peak picking method is useful as it is based on the resonance presented in FRF (Olmos and Roesset, 2010). To obtain the value of the $\zeta$ it is necessary to find the frequency of the maximum value of the resonance and the frequencies corresponding to the values of $A_{\max } / \sqrt{2}$. Using equation presented in Fig. 2 it is possible to get damping ratio of the system. To obtain structural damping ratio damping and stiffness resonance occurring in the FRF have to be approximated with use of the circle fit method. The Modified Least Square Method is very accurate and uses simple function, easy to implement in the software (Umbach and Jones, 2003). 
On base of the approximated circle it is possible to get the desired values of $\eta, C$ and K. Fig. 3 presents how structural damping can be obtained from the circle fitted to the experimental data.

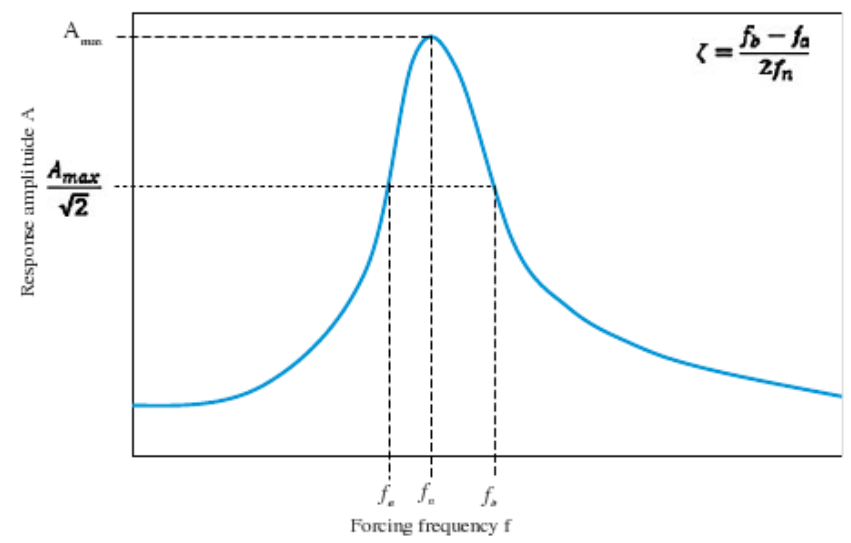

Fig. 2. Peak picking method for obtaining damping ratio of the system from the FRF (Umbach and Jones, 2003)

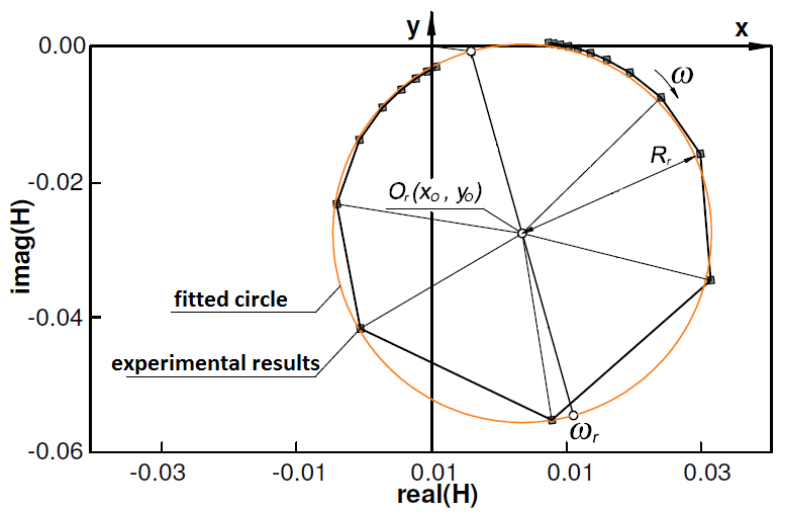

Fig. 3. The circle fit method for obtaining structural damping ratio, damping and stiffness of the system from the FRF (Maia, 1999), where $O_{r}$ is a center, $R_{r}$ is radius, $\omega_{r}$ is frequency of the resonsce, $\omega$ is increasing frequency of fitted circle

\section{DESIGN CONCEPT OF THE TEST STAND}

To design desired test stand some issues have to be taken into consideration to assure its proper work. First of all, as it was mentioned in previous section the test stand for determining properties of the MRE should have only one degree of freedom. Such situation happens only in the simulations and is used for simplification of the calculations. Real systems have many degrees of freedom, however this disadvantage can be overcome. Therefore the construction have to work as it would have only one degree of freedom with minimal reactions in other degrees of freedom.

Second important aspect of the test stand is that it have to be nonmagnetic as it will work with strong magnetic fields acting on the MRE material and on the construction of the test stand. Any magnetic element in the test stand that would be attracted or repelled by magnetic field generators would interfere with the test results, therefore the test stand have to be made out of nonmagnetic materials.

Third aspect that have to be considered is vibration isolation of the test stand, as external vibrations can interfere with the test results. The test stand should not require any specialized construction like floating floor that would mitigate the external vibrations originated out of the test stand.

\subsection{Construction}

The construction of the test stand should work as it has only one degree of freedom, therefore two plates placed one on top of another with tested material in between should work fine. Such solution will allow the MRE material to work in shear in the direction of the excitation (Giraudeua and Pierron, 2005). Such solution allows various setups of the MRE samples in the test stand. Thanks to such approach not only parameters connected with the material itself but also with shape, size and number of samples used in the experiment can be obtained. To mitigate external vibrations lower plate can be suspended on the elastic bands what should isolate the test stand from the vibrations not originated in the test stand. In such case test stand will behave as pendulum and during the experiment it will oscillate, therefore mass of whole system have to be big enough so the frequency of the oscillation will be below $1 \mathrm{~Hz}$. The idea for such test stand is presented in the Fig. 4. The lower plate M1 would be hanged in the air to isolate it form the external vibrations and to minimalize effects of its own vibrations. Also the mass of plate M1 should be much greater than that of plate M2 to move part of the FRF corresponding to plate M1 toward zero what would separate peaks visible on frequency response function.

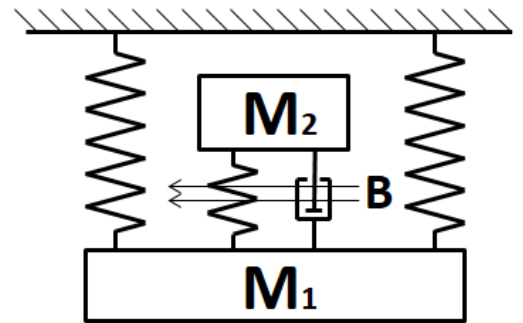

Fig. 4. Scheme of the concept of the test stand for determining damping of the magnetorheological elastomers, where $\mathrm{M}_{1}$ is lower plate, $M_{2}$ is upper plate, $B$ is magnetic field

The shape of the tested MRE should be cylindrical as such shape will allow to uniformly expose it to the magnetic field. Number of the test samples used simultaneously for testing depend on the need and can vary from one to many, however at least three test samples will guaranty stability of the upper plate M2 and will prevent it from rocking from side to side.

As the test stand will work in high magnetic field all elements have to be nonmagnetic, in such case stone (i.e.: granite or marble) would work perfect for both plates. In such case there would be no problem with obtaining high masses of both plates. All other elements of the test stand will have to be made out of other nonmagnetic and nonconductive materials as eddy currents can also effect the tests.

\subsection{Magnetic field source}

Magnetorheological elastomers require source of the variable magnetic field to work as adjustable material. The magnetic field 
can be applied to the material using few approaches: magnetic coils, permanent magnets or controllable magnetic matrix. Magnetic field that will stimulate MRE material should be uniform in the area where material will work and easy to change. Permanent magnets does not meet those requirements on the other hand magnetic coils can create easy to change uniform field however they would have large dimensions if generated field would have to be more than couple of $\mathrm{mT}$. The solution is magnetic matrix that is small and can generate magnetic field over $100 \mathrm{mT}$ with possibility for easy adjustment. Fig. 5 presents various ring magnetic matrices known as Halbach arrays. Depending on the setup of magnets creating the array it can create different shape of magnetic field inside the ring.
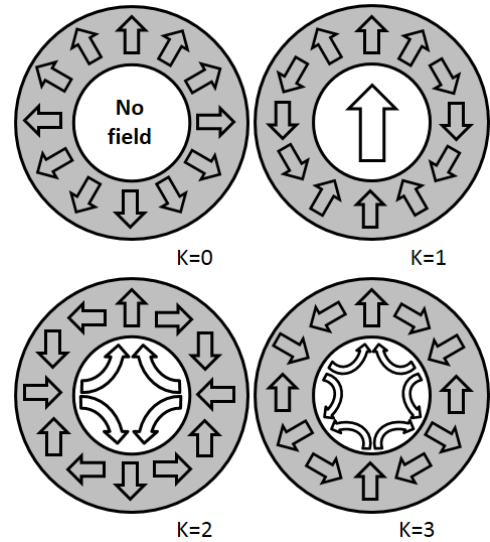

Fig. 5. Possible setups of round Halbach arrays that generate magnetic field in specyfic direction, where $\mathrm{K}=0, \mathrm{~K}=1, \mathrm{~K}=2, \mathrm{~K}=3$ are types of magnet setups. $K$ represents number of phases of the array

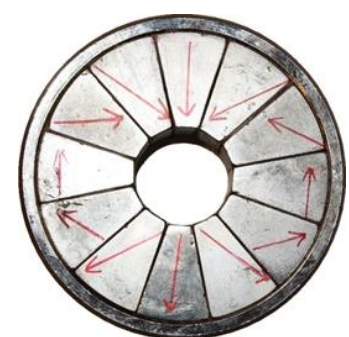

Fig. 6. Example of Halbach magnetic matrix with $\mathrm{K}=1$
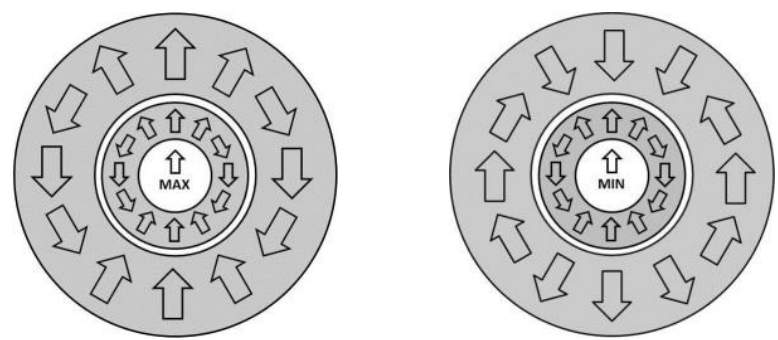

Fig. 7. Setup of magnets in double round Halbach array for obtaining minimum and maximum magnetic field inside of it

Fig. 6 presents an example of the round Halbach array with $\mathrm{K}=1$. This specific array creates uniform magnetic field inside of it. However to have possibility to change the value of magnetic field inside the matrix there is an idea to place two or more round Halbach arrays around one another and rotate them around each other to change magnetic field inside (Choi and Yoo, 2008). In the two-ring Halbach array change of the angle of the outer element from $0^{\circ}$ to $180^{\circ}$ causes change from maximum to minimum value of the created magnetic field. To determine maximal and minimal values of magnetic field inside the double matrix simulation using FEMM software was created, Fig. 7 presents setup of magnets in double array.

Fig. 8 presents extreme positions of the magnetic matrix. FEMM software allows to create only $2 \mathrm{D}$ simulations therefore the device was simulated in most useful view which is from the top. Magnets used in the simulations were square $7 \times 7 \mathrm{~mm} \mathrm{NdFeB}$ N40 neodymium magnets, and the opening inside had diameter of $25 \mathrm{~mm}$. The inner element was constructed out of 12 magnets and outer one out of 20 neodymium magnets. Simulation indicated that the maximum magnetic field that can be obtained using such system of magnets can create inside almost uniform magnetic field with maximum absolute value of about $650 \mathrm{mT}$ and minimum of about $100 \mathrm{mT}$ what gives a change in the value of the absolute magnetic field of $550 \mathrm{mT}$.

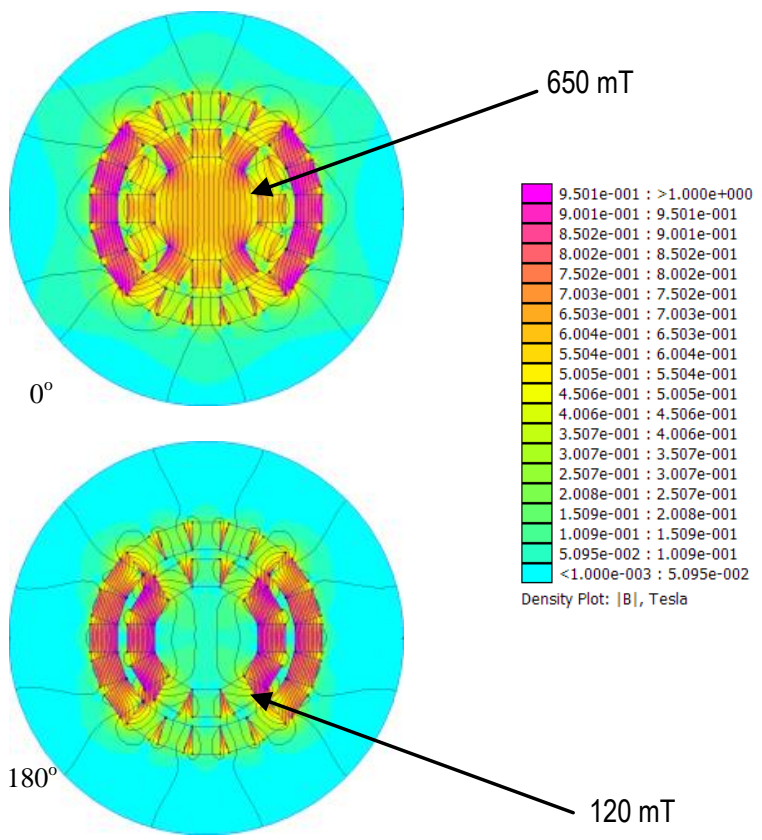

Fig. 8. Simulation of double Halbach magnetic matrix with outer part rotated with respect to the inner part what changes generated magnetic field

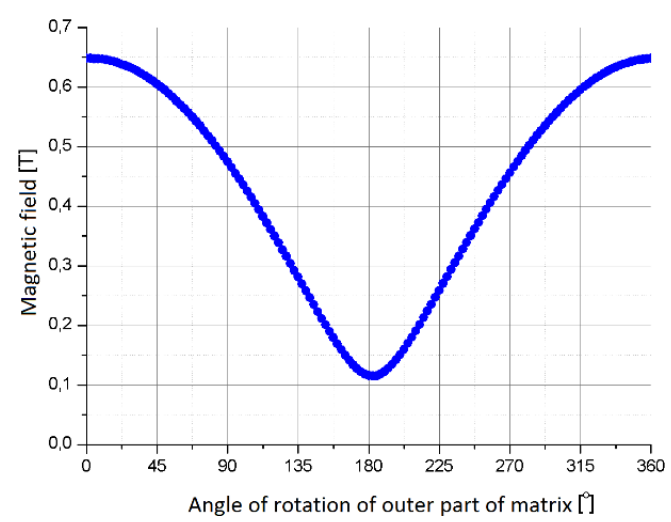

Fig. 9. Change of the magnetic field inside double Halbach matrix with the change of the angle of the outer element

The simulation was also performed for the change of the angle of the outer array by $2.5^{\circ}$ and the values of the absolute mag- 
netic field in tesla [T] in the center point was read. Graph in Fig. presents how changes the absolute value of magnetic field inside the double Halbach array with the change of the angle. In Fig. 9 is presented graph of the absolute value of magnetic field in the center of the double round Halbach array versus the angle of rotation of the outer part of the array.

\subsection{Sketch of the test stand}

On bases of the previous assumptions a sketch of the test stand was prepared. The sketch presents the setup of all of the elements and signals that will be present in the real test stand. Model presents two stone plates with MRE material placed in between them with round double Halbach arrays around them. At one end of the upper plate accelerometer is placed that will be collecting data from the upper plate, on the other side of that plate there is a place that will be excited using impact hammer. Both accelerometer and impact place are aligned and in the symmetry line of the setup. The double Halbach arrays that surround the MRE cylinders are placed on the bottom of the sample and on the top of the lower plate to ensure its vibrations will not interfere with the upper plates vibrations. The hanging of the test stand is presented only symbolically. Fig. 10 presents the model with the description of all the elements.

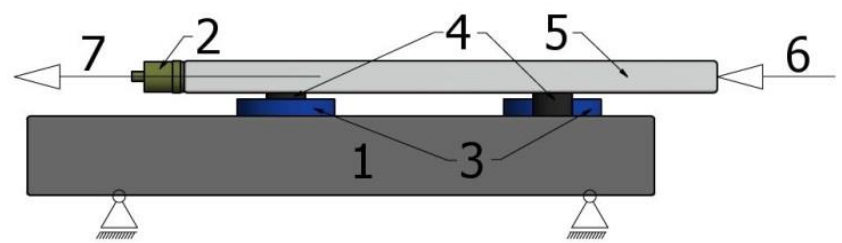

Fig. 10. Sketch of the test stand for determining properties of magnetorehological elastomers prepared using Autodesk Inventor software, where: 1 - lower plate; 2 - accelerometer; 3 - double Halbach arrays; 4 - MRE samples; 5 - upper plate; 6 - exitation signal from impact hammer; 7 - response signal

\section{SUMMARY}

The design concept of test stand presented in the article covers all aspects of the design process of the desired test stand. The basic idea of the test stand is to utilize the principle of modal analysis in the test stand for examining damping properties of magnetorheological elastomers. For this purpose single degree of freedom system was adapted to increase the effectiveness of the test stand and reduce interferences that might appear during the test. The great advantage of the one degree of freedom test stand is that it presents single result that can be easily interpreted and analyzed using the peak picking method and the circle fit method.

As the stand requires use of strong magnetic field all its elements were designed as nonmagnetic and nonconductive so the magnetic field would not distort the results. Also the external vibrations would strongly interfere with the results therefore design provides suspension for the test stand such that will eliminate all the effects related to the external vibrations.

The magnetic field presented in the article is a promising idea of mechanically controlled magnetic array that creates uniform magnetic field. The double round Halbach array presented in the article have been adapted for the demanding conditions of the test stand including small size of the device, ease of the control and creation of relatively high magnetic field and significant change in the created magnetic field. The array have been also modeled using FEMM software.

All those aspects were joined together and the model of the test stand have been prepared to present how it will be constructed and how it will operate. On base of this design concept the real test stand will be constructed according to the specification presented in the article. The results obtained using the test stand will help to designing working and applicable vibration dampers based on the MRE material.

\section{REFERENCES}

1. Choi J. S., Yoo J. (2008), Design of a Halbach Magnet Array Based on Optimization Techniques, Magnetics, 44, 10, 2361-2366.

2. Giraudeau A., Pierron F. (2005), Identification of stiffness and damping properties of thinisotropic vibrating plates using the virtual fields method: theory and simulations, Journal of Sound and Vibration, 284, 757-781.

3. H. Raich and P. Blümler (2004), Design and construction of a dipolar halbach array with a homogeneous field from identical bar magnets: Nmr mandhalas. Concepts in Magnetic Resonance Part B: Magnetic Resonance Engineering, 23B (1):16-25.

4. I. Kasa (1976), A circle fitting procedure and its error analysis, IEEE Trans. Instrum. Meas., Vol. 25, 8-14.

5. Kaleta J., Królewicz M., Lewandowski D. (2011), Magnetomechanical properties of anisotropic and isotropic magnetorheological composites with thermoplastic elastomer matrices, Smart Materials \& Structures, 20, 1-12.

6. Liao G. J., Gong X. L., Kang C. J., Xuan S. H. (2011), The design of an active-adaptive tuned vibration absorber based on magnetorheological elastomer and its vibration attenuation performance, Smart Materials and Structures, 20:75015-75024.

7. Maia N. M. M. (1999), Modal Identification Methods in the Frequency Domain, Modal Analysis and Testing, 363, 251-264.

8. Olmos B. A., Roesset J. M. (2010), Evaluation of the half-power bandwidth method to estimate damping in system without real modes, Earthquake Engineering and Structural Dynamics, 39, 1671-1686.

9. Umbach D., Jones K. (2003), A Few Methods for Fitting Circles to Data, Instrumentation and Measurement, 52, 1881-1885.

10. Zhu Z. Q., Howe D. (2001), Halbach permanent magnet machines and applications: a review, IEE Proceedings - Electric Power Applications, Vol. 148, No. 4, 299-308.

This research was partly supported by Wroclaw Research Centre EIT + within the project 'The Application of Nanotechnology in Advanced Materials' -NanoMat (POIG.01.01.02-02-002/08) financed by (IInnovative 